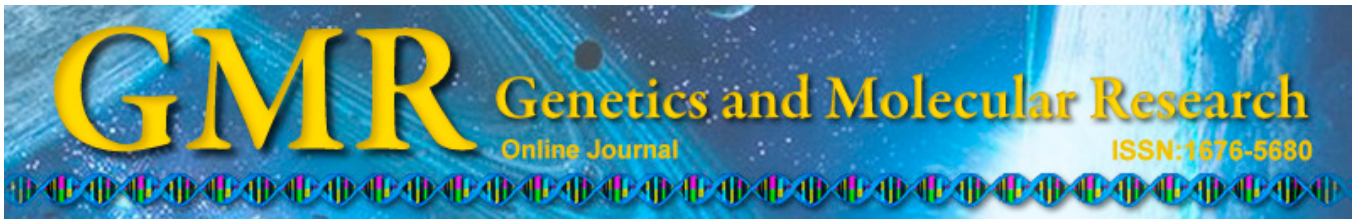

\title{
Clinical features and treatment of endophthalmitis after cataract surgery
}

\author{
J. Zhu' ${ }^{1}$ and Z.H. $\mathbf{L i}^{2}$ \\ ${ }^{1}$ Department of Ophthalmology, \\ ${ }^{2}$ Department of Ophthalmology, \\ Corresponding author: Z.H. Li \\ E-mail: zhaohuilicn@126.com \\ Genet. Mol. Res. 14 (2): 6617-6624 (2015) \\ Received May 22, 2014 \\ Accepted March 4, 2015 \\ Published June 18, 2015 \\ DOI http://dx.doi.org/10.4238/2015.June.18.4
}

The First Affiliated Hospital of Chinese PLA General Hospital, Beijing, China

PLA General Hospital of Beijing Haidian District, Beijing, China

\begin{abstract}
The aim of this study was to investigate the clinical features and treatment results of endophthalmitis after cataract surgery. Five patients with endophthalmitis after phacoemulsification with intraocular lens implantation were enrolled in this study. The pathogenesis, clinical manifestation, and surgical outcomes of 5 patients were compared. Three patients were surgically treated with anterior chamber irrigation and vitrectomy with intravitreal injection. The remaining two patients were medically treated with an intravitreal injection of vancomycin and ceftazidime. Treatment results of the five patients were analyzed. Four patients had positive cultures for bacteria (two cases Staphylococcus epidermidis, one case Enterococcus faecalis, and one case head-like Staphylococcus). The culture of the fifth patient did not have bacterial growth. One year following treatment, four patients had restored visual acuity and a clear vitreous cavity. Retinal detachment and other complications were not observed. The remaining patient had a visual acuity of index at $30 \mathrm{~cm}$ one year following treatment. For patients with endophthalmitis after cataract surgery, a biochemical laboratory examination should be promptly performed
\end{abstract}


and should include a bacterial culture and drug sensitivity test. When necessary, vitrectomy combined with an intravitreal injection of vancomycin should be performed to treat the infection early and to help retain useful vision.

Key words: Cataract; Clinical feature; Endophthalmitis; Treatment

\section{INTRODUCTION}

Infectious endophthalmitis is the most serious complication that can occur following cataract extraction and intraocular lens (IOL) implantation. In fact, this intraocular infection can threaten visual acuity and, in some cases, even the patient's life. Previous studies indicate that the incidence of endophthalmitis has decreased since cataract surgery became a common procedure in the 1990s (Al-Mezaine et al., 2009). However, the rapid progression and accompanying poor prognosis of infectious endophthalmitis must be stressed by surgeons to their patients. Once it occurs, endophthalmitis can profoundly affect visual function and ocular tissues. Timely and effective therapy is extremely important to clinical prognosis and surgical treatment outcomes (Pijl et al., 2010). Here, we report the outcomes of using vitrectomy in combination with intravitreal injection to treat endophthalmitis occurring after phacoemulsification and IOL implantation.

\section{MATERIAL AND METHODS}

\section{Subjects}

Five patients who previously underwent phacoemulsification and IOL implantation were admitted to our hospital between May 2008 and December 2011 for endophthalmitis. Three male ( 3 eyes) and two female ( 2 eyes) patients between the ages of 41 and 79 years $(65.8 \pm 0.5$ years $)$ were included. Three patients underwent vitrectomy in combination with intravitreal vancomycin injection. The study conduct adhered to the tenets of the Declaration of Helsinki. Additionally, the study was conducted with approval from the Ethics Committee of PLA General Hospital of Beijing Haidian District. Written informed consent was obtained from all participants.

\section{Surgical procedures}

All five patients presented with endophthalmitis after undergoing conventional phacoemulsification combined with IOL implantation at another hospital. All patients were admitted to our hospital for further treatment, which included either intravitreal injection (2 eyes) or vitrectomy and intravitreal injection combination therapy ( 3 eyes).

\section{Clinical presentation}

Patients presented with characteristic endophthalmitis symptoms (e.g., acute vision decrease of varying degrees, red eye, ophthalmalgia, etc.) between two and nine days following cataract surgery. Patient 1 had a red eye and ophthalmalgia nine days after surgery. Deci- 
mal visual acuity was 0.10 and slit-lamp examination revealed bulbar conjunctival hyperemia (+), mild corneal edema, and aqueous humor Tyn (++). Patient 2 also had evident ophthalmalgia four days after cataract surgery, at which point decimal visual acuity was 0.05. Slit-lamp examination revealed mixed bulbar conjunctival hyperemia $(++)$, corneal edema (+), and aqueous humor Tyn $(++)$. An ultrasound B-scan found no vitreous opacities. The white blood cell (WBC) count from a peripheral venous blood sample was 11.2 $\mathrm{x} 10^{8}$ cells/L. Patient 3 presented with a red eye and ophthalmalgia seven days following cataract surgery, at which point decimal visual acuity was hand move at $10 \mathrm{~cm}$. Slit-lamp examination revealed mixed bulbar conjunctival hyperemia $(+++)$, corneal edema $(++)$, and aqueous humor Tyn $(+++)$. An ultrasound B-scan showed vitreous opacities $(+)$ and a fibrous exudate on the IOL surface. A peripheral blood sample was analyzed and revealed a WBC count of $12.5 \times 10^{8}$ cells/L. Patient 4 presented with significant red eye, ophthalmalgia, and mild headache two days after cataract surgery, at which time visual acuity was hand motion at $10 \mathrm{~cm}$. Slit-lamp examination revealed mixed bulbar conjunctival hyperemia $(+++)$, corneal edema $(++)$, aqueous humor Tyn $(+++)$, and hypopyon. Additionally, the fluid surface was approximately $3 \mathrm{~mm}$ and fibrous exudate was observed on the IOL surface. An ultrasound B-scan showed a vitreous opacity $(++)$. The patient also had an elevated body temperature of $39^{\circ} \mathrm{C}$ and a peripheral WBC count of $13.8 \times 10^{8}$ cells/L. Patient 5 presented with ophthalmalgia and a red eye eight days following cataract surgery, at which time visual acuity was finger index at $10 \mathrm{~cm}$. Slit-lamp examination revealed mixed bulbar conjunctival hyperemia $(+++)$, corneal edema $(++)$, aqueous humor Tyn $(+++)$, and hypopyon. Additionally, the fluid surface was approximately $1 \mathrm{~mm}$ and fibrous exudate was observed on the IOL surface. An ultrasound B-scan showed a vitreous opacity $(+)$. The patient also had an elevated body temperature of $38.5^{\circ} \mathrm{C}$ and a peripheral WBC count of $12.8 \times 10^{8}$ cells $/ \mathrm{L}$.

\section{Patient general health}

One patient suffered from diabetes and hypertension for 15 years, which was complicated by 3 years of renal insufficiency. Two patients had diabetes for 3 years. One patient had systemic rheumatoid arthritis for 8 years. The remaining patient had coronary atherosclerosis.

\section{Clinical treatment}

Based on primary disease signs and symptoms, 3 of 5 cases had anterior chamber irrigation before posterior vitrectomy, which was followed by intravitreal injection of $1 \mathrm{mg}$ vancomycin and $2.25 \mathrm{mg}$ ceftazidime. Samples of aqueous humor and the vitreous were collected for bacterial culture before the intravitreal injection was given. Patients were also treated systemically ( $1 \mathrm{~g}$ vancomycin through intravenous drip, twice a day) and topically $(0.5 \%$ levofloxacin and $1 \%$ atropine eye drops, two to three times a day). Treatments were adjusted based on bacterial culture and drug sensitivity testing results. In addition, one patient received a periocular antibiotic injection $(5 \mathrm{mg} / 0.5 \mathrm{~mL}$ vancomycin and $10 \mathrm{mg} / 0.5 \mathrm{~mL}$ ceftazidime $)$ and one patient received an antibiotic intravitreal injection $(1 \mathrm{mg} / 0.1 \mathrm{~mL}$ vancomycin and 2.25 $\mathrm{mg} / 0.1 \mathrm{~mL}$ ceftazidime), a systemic antibiotic injection, $1 \mathrm{~g}$ vancomycin two times a day, $1 \mathrm{~g}$ ceftazidime three times a day, and $40 \mathrm{mg}$ intravenous methylprednisolone once a day. 


\section{RESULTS}

\section{Microbiological examination}

All patients underwent bacterial culture and drug sensitivity testing on aqueous humor and vitreous fluid samples. Two patients had culture-negative vitreous and culture-positive aqueous. One positive culture grew Enterococcus faecalis that was sensitive to penicillin and the other grew head-like Staphylococcus that was sensitive to rocephin and ciprofloxacin. Two patients had culture-positive vitreous and culture-negative aqueous humor. Both positive cultures grew Staphylococcus epidermidis that was sensitive to vancomycin. The final patient had culture-negative vitreous and aqueous humor (Figure 1).

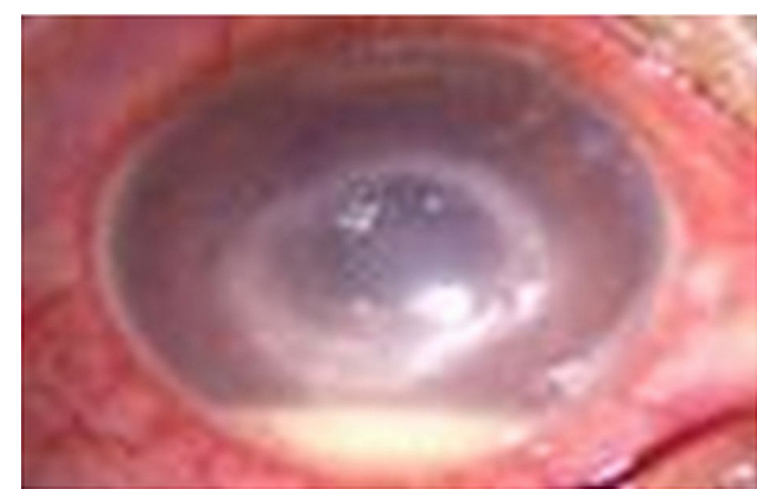

Figure 1. One patient with diabetes and renal insufficiency presented with hypopyon at day 2 after cataract surgery.

\section{Treatment Efficacy}

In this study, all symptoms of endophthalmitis were significantly alleviated following treatment. Additionally, intraocular pressure was well-maintained. Two eyes that presented with hypopyon had absorbed anterior chamber fibrous exudate, resolved hypopyon, and aqueous humor Tyn $(+)$. Aqueous flare in the anterior chamber and vitreous opacities were not observed in the other patients. Four cases had restoration of visual acuity, but the remaining patient had a final visual acuity of index at $30 \mathrm{~cm}$ (Table 1).

Table 1. Treatment of endophthalmitis post-cataract operation.

\begin{tabular}{llclll}
\hline No/Gender/Age & Cataract surgery & $\begin{array}{c}\text { Days between cataract } \\
\text { surgery and onset }\end{array}$ & Microbiological examination & Preoperative visual acuity & $\begin{array}{l}\text { Postoperative } \\
\text { visual acuity }\end{array}$ \\
\hline $1 /$ male/41 & PHACO + IOL & 9 & Head-like Staphylococcus & 0.1 & 0.8 \\
$2 / \mathrm{female} / 65$ & PHACO + IOL & 4 & Staphylococcus epidermidis & 0.05 & 0.5 \\
$3 / \mathrm{male} / 69$ & PHACO + IOL & 7 & Staphylococcus epidermidis & Hand movement $/ 10 \mathrm{~cm}$ & 0.3 \\
$4 / \mathrm{male} / 75$ & PHACO + IOL & 2 & Enterococcus faecalis & Hand movement $/ 10 \mathrm{~cm}$ & Index $/ 30 \mathrm{~cm}$ \\
$5 / \mathrm{female} / 79$ & PHACO + IOL & 8 & Undetected & Index $/ 10 \mathrm{~cm}$ & 0.2 \\
\hline
\end{tabular}

\section{DISCUSSION}

Recent incidence of endophthalmitis following cataract surgery has been markedly 
reduced because of improvements in ophthalmological microscopy and sterile surgical procedures. In general, endophthalmitis that occurs following cataract extraction and IOL implantation tends to be classified as acute or chronic, depending on the pathogenic bacteria virulence. Acute endophthalmitis has an early onset $(<1$ week after surgery), rapid progression, and a severe illness state. Chronic endophthalmitis occurs with lower virulent bacteria and has a late onset, typically several weeks or months after surgery (Miller et al., 2008). In this study, two patients developed endophthalmitis two and four days after cataract surgery, which was regarded as acute endophthalmitis.

Diabetes, upper respiratory tract infection, and renal insufficiency are systemic factors known to cause infection (Fintelmann and Naseri, 2010). Previous findings (Li et al., 2004) suggest that age, gender, and race are closely associated with the incidence of endophthalmitis, which increases in patients older than 80 years and occurs 1.83 times more often in patients older than 90 years. Although some reports (Bron and Creuzot-Garcher, 2007; Díez et al., 2009) indicate a potential association between endophthalmitis incidence and intraoperative aqueous humor bacteria, we are not convinced that the presence of the microbe is the pathogenic bacteria responsible for endophthalmitis. Therefore, it is possible that the aqueous humor has some innate antibacterial qualities. Endophthalmitis may occur when a patient's immune resistance is weakened or when physical defense mechanisms have been disrupted. Previous studies have reported a higher incidence of endophthalmitis in diabetic patients than in their non-diabetic counterparts following cataract surgery, regardless of whether an IOL was implanted or not (Teoh et al., 2005). Because the blood-aqueous humor barrier is vulnerable to injury, especially in the presence of capillary dysfunction, a later onset or relapse of endophthalmitis is likely to occur. The three diabetic patients included in our study had stable blood glucose levels, but still had a significantly lower resistance than those patients without systemic complications. This was especially true for patients with renal insufficiency, who presented with postoperative endophthalmitis earlier than the other subjects (Table 1).

Blepharitis, conjunctivitis, lacrimal canaliculus dacryocystitis, and lacrimal duct obstruction are primary topical factors that can lead to infection. The human conjunctival sac is normally open and has a large number of meibomian glands and accessory lacrimal gland open. Approximately two-thirds of normal subjects have microflora in their conjunctival sacs, and the conjunctival sac and ocular adnexa are the most common sources of infection. Thus, antibiotics eye drops were not administered before surgery or were given for an insufficient period. Therefore, this may be a vital reason for the incidence of postoperative endophthalmitis. After thorough patient interviews, we discovered that two patients suffered from chronic lacrimal duct obstruction and chronic blepharitis. These patients also used antibiotic eye drops for less than 24 $\mathrm{h}$ before cataract surgery. One of these patients was diabetic, was discharged $24 \mathrm{~h}$ after surgery, and failed to use antibiotic eye drops in the prescribed manner after surgery.

Some studies have revealed that the incidence of endophthalmitis was $0.31 \%$ after extracapsular cataract extraction, and only $0.07 \%$ following phacoemulsification. This suggests that surgical incision size positively correlates with the probability of pathogenic bacteria entering the eye (Mayer et al., 2003). Additionally, some investigators have reported an elevated incidence of endophthalmitis by more than $300 \%$ when a scleral tunnel incision was replaced with a transparent corneal incision during cataract surgery. Therefore, incision type and size are major factors in increasing postoperative endophthalmitis incidence (Lundström et al., 2007; Anijeet, 2010). Lundstrom (2006) found no convincing evidence to confirm the association between transparent corneal incision use and endophthalmitis incidence. However, 
transparent corneal incision use may still play a role in the occurrence of endophthalmitis under some circumstances. Nevertheless, it has been widely accepted that neither transparent corneal incisions nor scleral tunnel incisions increase the risk for endophthalmitis when incision size is within $3 \mathrm{~mm}$ (Lalwani et al., 2008). However, incisional leaks and poor healing may be risk factors for postoperative endophthalmitis (Chee et al., 2010). One day following cataract surgery, $45 \%$ of patients had positive bacterial cultures. Therefore, early eye care after surgery is of great significance (García-Sáenz et al., 2010).

The vitreous is an avascular tissue and removes metabolites at a low rate. Therefore, it can be a suitable medium for bacteria and other microbes, which are likely to multiply when present. Anterior chamber irrigation in combination with vitrectomy is effective in treating acute cases of endophthalmitis (Endophthalmitis Study Group and ESCRS, 2007). Together, these treatments eliminate pathogenic bacteria, bacterial toxin, inflammatory exudates, and necrotic tissues, while completely disrupting the favorable microenvironment for bacteria. This therapy, in combination with intravitreal injection, also attains high-dose efficacy in treating topical lesions. In our study, patients underwent vitrectomy and intravitreal injection immediately after being diagnosed with endophthalmitis. The two patients that underwent combined therapy had better visual acuity than those receiving injection alone. This indicates that patients with severe disease and poor systemic health should undergo immediate vitrectomy instead of waiting for visual acuity to decrease to light perception. In addition, when patients have endophthalmitis for a long time, bacterial toxins may attack the retina and other ocular structures, leading to permanent, severe vision loss. Visual acuity improved by at least two lines in 4 of 5 eyes (80\%) following vitrectomy. One patient with diabetes and hypertension complicated by renal insufficiency presented with the signs and symptoms of acute endophthalmitis, including acute onset, rapid progression, and a severe disease course. After undergoing combination therapy with vitrectomy, repeated anterior chamber irrigation, and intravitreal injection, the patient's visual acuity did not significantly improve. However, endophthalmitis symptoms were controlled, suggesting that early vitrectomy and intraocular drug injection can improve prognosis and treatment efficacy. This can be accomplished even in the case of severe injury in patients with systemic disorders and poor visual acuity (Arbisser, 2008).

Endophthalmitis treatment principles are widely recognized and are based upon the severity of visual loss induced by the condition. The first phase is defined as a slight opacity of the anterior chamber with no hypopyon or vitreous opacity. Patients in this phase should be monitored every 4 to $6 \mathrm{~h}$, unless progression is rapid, in which case patients should be monitored every $2 \mathrm{~h}$. Anterior chamber irrigation with antibiotics should be performed when necessary, but an aqueous humor sample should be collected for bacterial culture and drug sensitivity testing prior to irrigation. Irrigation solutions generally contain $0.02 \mathrm{mg} / \mathrm{mL}$ vancomycin and 0.04 $\mathrm{mg} / \mathrm{mL}$ ceftazidime diluted in phosphate buffered saline. Before the irrigation procedure, $1 \mathrm{~mL}$ irrigation solution was added to $500 \mathrm{~mL}$ ophthalmic balanced salt solution for use in anterior chamber irrigation. In the second phase, hypopyon was observed, but no vitreous opacity was noted. Anterior chamber irrigation combined with intravitreal drug injection was performed. The optimal drugs for intravitreal injection are thought to be vancomycin $(1 \mathrm{mg} / 0.1 \mathrm{~mL})$ with ceftazidime $(2.00-2.25 \mathrm{mg} / 0.1 \mathrm{~mL})$ or vancomycin $(1-2 \mathrm{mg} / 0.1 \mathrm{~mL})$ with amikacin $(0.4 \mathrm{mg} / 0.1$ $\mathrm{mL}$ ). Aqueous humor and vitreous fluid were sampled for bacterial culture and drug resistance testing before the antibiotic solutions were administered via intravitreal injection. In the third phase, both hypopyon and vitreous opacities occurred. Vitrectomy combined with intravitreal injection was used to treat the endophthalmitis. The disease course should be monitored every 4 
to $6 \mathrm{~h}$ to identify the disease stage and adjust treatments accordingly.

Preventive measures should be considered and taken before, during, and after cataract surgery. Before surgery, the infectious eyelid, conjunctiva, lacrimal duct, and ocular adnexa should be treated. Endophthalmitis should be largely preventable, especially in patients with systemic diseases, such as diabetes (O'Brien et al., 2007). However, as a precaution, risk factors of postoperative infection should be comprehensively evaluated for each patient. Antibiotics eye drops given three days before surgery can help to minimize endophthalmitis. Lastly, the lacrimal duct and conjunctival sac should be thoroughly irrigated, especially the fornix, palpebral conjunctiva, and medial and lateral canthi.

Intraoperative preventive measures can also be helpful. Before making any incisions, the area from the eyebrow to the nose bridge midline on the operative side should be sterilized and isolated with an aseptic film. This avoids soaking the hole-towel. Additionally, surgical skills should be strengthened and surgical time should be shortened. The IOL should remain wrapped until usage and unwrapped under aseptic conditions. This will reduce its exposure time to the surroundings (Patwardhan et al., 2006).

Preventive measures can also be taken after surgery to minimize the risk of postoperative infections (Friling et al., 2013). Patients and their families should be educated after surgery on how to protect operative eyes and prevent wound infection. It should be stressed that eye drops need to be administered at the given time and that eye drops used before and after surgery should be separated. Ocular sample testing and combined vitrectomy and intravitreal injection should be performed when infection is suspected. These measures will help to control disease progression and improve the chances of restoring visual acuity.

\section{REFERENCES}

Al-Mezaine HS, Kangave D, Al-Assiri A and Al-Rajhi AA (2009). Acute-onset nosocomial endophthalmitis after cataract surgery: incidence, clinical features, causative organisms, and visual outcomes. J. Cataract Refract. Surg. 35: 643-649. Anijeet D (2010). Endophthalmitis after cataract surgery. Ophthalmology 117: 853-855.

Arbisser LB (2008). Safety of intracameral moxifloxacin for prophylaxis of endophthalmitis after cataract surgery. $J$. Cataract Refract. Surg. 34: 1114-1120.

Bron A and Creuzot-Garcher C (2007). Prophylaxis for postcataract endophthalmitis. J. Fr. Ophtalmol. 30: 1027-1036.

Chee SP, Ti SE, Lim L, Chan AS, et al. (2010). Anterior segment optical coherence tomography evaluation of the integrity of clear corneal incisions: a comparison between 2.2-mm and 2.65-mm main incisions. Am. J. Ophthalmol. 149: 768-776.

Díez MR, De la Rosa G, Pascual R, Girón C, et al. (2009). Prophylaxis of postoperative endophthalmitis with intracameral cefuroxime: a five years' experience. Arch. Soc. Esp. Oftalmol. 84: 85-89.

Endophthalmitis Study Group and European Society of Cataract and Refractive Surgeons (2007). Prophylaxis of postoperative endophthalmitis following cataract surgery: results of the ESCRS multicenter study and identification of risk factors. J. Cataract Refract. Surg. 33: 978-988.

Fintelmann RE and Naseri A (2010). Prophylaxis of postoperative endophthalmitis following cataract surgery: current status and future directions. Drugs 70: 1395-1409.

Friling E, Lundström M, Stenevi U and Montan P (2013). Six-year incidence of endophthalmitis after cataract surgery: Swedish national study. J. Cataract Refract. Surg. 39: 15-21.

García-Sáenz MC, Arias-Puente A, Rodríguez-Caravaca G, Andrés Alba Y, et al. (2010). Endophthalmitis after cataract surgery: epidemiology, clinical features and antibiotic prophylaxis. Arch. Soc. Esp. Oftalmol. 85: 263-267.

Lalwani GA, Flynn HW Jr, Scott IU, Quinn CM, et al. (2008). Acute-onset endophthalmitis after clear corneal cataract surgery (1996-2005). Clinical features, causative organisms, and visual acuity outcomes. Ophthalmology 115: 473-476.

Li J, Morlet N, Ng JQ, Semmens JB, et al. (2004). Significant non-surgical risk factors for endophthalmitis after cataract surgery: EPSWA fourth report. Invest. Ophthalmol. Vis. Sci. 45: 1321-1328.

Lundstrom M (2006). Endophthalmitis and incision construction. Curr. Opin. Ophthalmol. 17: 68-71.

Lundström M, Wejde G, Stenevi U, Thorburn W, et al. (2007). Endophthalmitis after cataract surgery: a nationwide prospective study evaluating incidence in relation to incision type and location. Ophthalmology 114: 866-870. 
Mayer E, Cadman D, Ewings P, Twomey JM, et al. (2003). A 10 year retrospective survey of cataract surgery and endophthalmitis in a single eye unit: injectable lenses lower the incidence of endophthalmitis. Br. J. Ophthalmol. 87: 867-869.

Miller JJ, Scott IU, Flynn HW Jr, Smiddy WE, et al. (2008). Endophthalmitis caused by Bacillus species. Am. J. Ophthalmol. 145: 883-888.

O’Brien TP, Arshinoff SA and Mah FS (2007). Perspectives on antibiotics for postoperative endophthalmitis prophylaxis: potential role of moxifloxacin. J. Cataract Refract. Surg. 33: 1790-1800.

Patwardhan A, Rao GP, Saha K and Craig EA (2006). Incidence and outcomes evaluation of endophthalmitis management after phacoemulsification and 3-piece silicone intraocular lens implantation over 6 years in a single eye unit. $J$. Cataract Refract. Surg. 32: 1018-1021.

Pijl BJ, Theelen T, Tilanus MA, Rentenaar R, et al. (2010). Acute endophthalmitis after cataract surgery: 250 consecutive cases treated at a tertiary referral center in The Netherlands. Am. J. Ophthalmol. 149: 482-487.

Teoh SC, Lee JJ, Chee CK and Au Eong KG (2005). Recurrent Enterococcus faecalis endophthalmitis after phacoemulsification. J. Cataract Refract. Surg. 31: 622-626. 